\title{
Comparative Analysis of Saline Sonohysterosalpingography to Hysterosalpingography in the Diagnosis of Utero-Tubal Pathology amongst Infertile Women at the University of Port Harcourt Teaching Hospital
}

\author{
E. W. Nnah ${ }^{1}$, V. K. Oriji ${ }^{1,2}{ }^{*}$, C. E. Agi ${ }^{2,3}$ \\ ${ }^{1}$ University of Port Harcourt Teaching Hospital, Port Harcourt, Rivers State, Nigeria \\ ${ }^{2}$ Department of Obstetrics and Gynaecology, College of Health Sciences, University of Port Harcourt, Rivers State, Nigeria \\ ${ }^{3}$ Department of Radiology, College of Health Sciences, University of Port Harcourt, Rivers State, Nigeria \\ Email: *vaduneme. oriji@uniport.edu.ng
}

How to cite this paper: Nnah, E.W., Oriji, V.K. and Agi, C.E. (2019) Comparative Analysis of Saline Sonohysterosalpingography to Hysterosalpingography in the Diagnosis of Utero-Tubal Pathology amongst Infertile Women at the University of Port Harcourt Teaching Hospital. Open Journal of Medical Imaging, 9, 58-68.

https://doi.org/10.4236/ojmi.2019.94006

Received: October 15, 2019

Accepted: December 27, 2019

Published: December 30, 2019

Copyright (๑) 2019 by author(s) and Scientific Research Publishing Inc. This work is licensed under the Creative Commons Attribution International License (CC BY 4.0).

http://creativecommons.org/licenses/by/4.0/

\begin{abstract}
Background: The evaluation of the uterus and its tubes cannot be overemphasized in the management of infertile women. Transvaginal saline sonohysterosalpingography (SHG) is another novel method with certain advantages for uterine and tubal assessment in women. Objective: To determine the accuracy of saline SHG findings by comparing it with conventional Hysterosalpingography (HSG) findings in infertile women. Result: The concordance between saline sonohysterosalpingography and hysterosalpingography for the diagnosis of submucous fibroids, uterine adhesions and tubal patency in this study was over $94 \%$. The saline SHG had sensitivity and specificity of $93.8 \%$ and $94.1 \%$ respectively for diagnosis of uterine fibroids (submucous) and a sensitivity and specificity of $62.5 \%$ and $98.9 \%$ in the diagnosis of intrauterine adhesions. It also had a sensitivity and specificity of $98.9 \%$ and $78.6 \%$ respectively in the detection of patent fallopian tubes. Conclusion: The ability of saline SHG to diagnose uterine pathology and tubal patency was comparable to that of conventional HSG. Saline SHG has the potential to replace HSG as the first-line test for the evaluation of uterine or tubal disease in infertile women.
\end{abstract}

\section{Keywords}

Saline Sonohysterosalpingography, Hysterosalpingography, Infertility 


\section{Introduction}

Infertility in women could be caused by disorders at the fallopian tubes, uterus, cervix, and ovaries. Specifically, the disorder of the fallopian tubes is the reason for infertility in about $35 \%-40 \%$ [1] [2]. The available methods for clinical evaluation of the endometrial cavity and tubal patency developed over time, include hysterosalpingography, transvaginal ultrasonography, saline sonohysterography, hystero-contrast sonography, hysteroscopy, and laparoscopy. Other investigations not readily employed for clinical evaluation of infertility include the CT and MRI [1] [2] [3] [4]. X-ray hysterosalpingography has been the main method used for the diagnosis of endometrial abnormalities and tubal patency in infertile women at the University of Port Harcourt Teaching Hospital as in other hospitals in Nigeria [5] [6] [7] [8]. HSG as a method is associated with complications such as pelvic pain, vomiting, and fever. Occasionally, patients react to the contrast media used. Patients' treatment could be delayed due to the time on the waiting list for HSG in the radiology department. Saline SHG is an imaging procedure in which isotonic saline is infused into the uterine cavity through the cervix to provide enhanced visualization of the endometrial cavity during transvaginal ultrasound examination [9]. Saline SHG uses saline infusion which is better tolerated and causes fewer side effects than the oil-based media used in HSG. The procedure can also be done during regular transvaginal ultrasound scan evaluation for infertility thereby reducing the time spent waiting for HSG before treatment can be offered. This novel method of transvaginal ultrasound and isotonic saline for assessing the uterine cavity and tubal patency was reported by Tüfekni et al. in 1992 [10]. However, saline enhanced endometrial visualization using abdominal ultrasound had earlier been reported by some other researchers [11] [12]. Saline SHG has been demonstrated in many studies to be superior to HSG in the diagnosis of submucous fibroids and endometrial polyps. Acholonu et al. and Goldberg et al. demonstrated that when saline SHG and HSG were compared against hysteroscopic diagnosis in two different independent studies, that saline SHG had a higher sensitivity and specificity in the diagnosis submucous fibroid [13] [14]. The diagnosis of tubal patency by saline SHG correlates positively with HSG diagnosis in about $93 \%$ of cases, and with laparoscopy diagnosis in $97 \%$ of cases [15]. Other researchers, such as Kumari et al. have reported a lower but comparable sensitivity and specificity of saline SHG in detecting tubal patency when compared to HSG [2].

Both procedures, saline SHG and HSG employ a similar technique. The main differences are the use of isotonic saline as the contrast medium and ultrasound waves for imaging in saline SHG instead of a radio-opaque contrast medium and $\mathrm{x}$-rays for the imaging in HSG. However, the two procedures produce similar results.

\section{Aims and Objectives}

The aim is to find a suitable alternative to HSG for women undergoing endome- 
trial cavity and tubal patency evaluation in infertile women.

The objective is to compare the findings from saline sonohysterosalpingography (saline SHG) to Hysterosalpingography (HSG) in the same group of infertile women.

\section{Materials and Methods}

A cohort study of 101 infertile women at the Gynecologic clinic of UPTH, from May 2016 to December 2016 was done. The sample size for the study was calculated using a simplified formula for calculating sample size for social studies research [16]. The sample size for this study was calculated using a simplified formula for calculating sample size $n=N / 1+N(e)^{2}$. Where $\mathrm{n}$ is the sample size. $\mathrm{N}$ is the study population of 120 (which was the average number of new cases of infertility seen at the gynaecological clinic over a period of six months). The margin of error, $d$ was put at 0.05 . The sample size was thus calculated to be 92 . An allowance for $10 \%$ attrition rate was added to the sample size for the study which gave 101 women. The ethics committee of the University of Port Harcourt Teaching Hospital gave ethical approval for the study. A written informed consent was signed by every participant before enrolment for the study. The procedures were explained to the patient before carrying it out. They were informed that opting out at any stage of the study will not affect the treatment of their conditions.

A questionnaire was administered to these patients before their investigations, to collect the following data: Age, parity, level of education attained, and period of infertility. HSG was performed during the proliferative phase of the menstrual cycle (on day ten of menses). The patient was placed in the lithotomy position, and a preliminary radiograph which is a plain radiograph of the pelvis was taken. A bivalve speculum was used to expose the cervix. The vagina and cervix were cleaned with chlorhexidine solution. The anterior lip of the cervix was grasped with a tenaculum; Leech Wilkinson cannula was introduced and stabilized to the cervix. Under fluoroscopic guidance, Devhexol 350 (johexol) a nonionionic low osmolar (osmolalility of $844 \mathrm{mos} / \mathrm{kg}$ ) contrast medium was introduced through the cannula into the uterus. The filling of the uterine cavity and the spillage from the fimbriae end was observed and documented with serial $\mathrm{x}$-ray films. The findings on HSG as to the presence of uterine myoma, uterine adhesions, and patency of fallopian tubes as reported by the radiologist were entered into an excel spreadsheet already containing the biodata from the questionnaire. A transvaginal saline SHG was done within 4 weeks of having done an HSG. A preliminary transvaginal ultrasonography was carried out. A size 6 Fr. Foleys catheter was passed through the external cervical ostium into the endometrial cavity and inflated with 2 millilitre of normal saline. The catheter is pulled outward to anchor the bulb at the internal cervical ostium. An assistant injects normal saline through the open channel of the Foleys catheter into the endometrial cavity in aliquots of 5 to 10 mllilitres whenever asked, as a second 
TVS is carried out. The endometrial cavity, fallopian tubes and the pouch of Douglas are studied during this procedure which lasts for about 7 - 10 minutes. The presence of a uterine fibroid and uterine adhesions were also noted. Next, the right and left fallopian tubes are examined for patency. The POD is also examined to confirm tubal patency. The findings from the saline HSG as reported by the gynecologist was then entered into the existing spreadsheet. Statistical analysis of the data was done with SPSS for windows version 20.0. and the result presented below.

EXCLUSION CRITERIA: This includes women who withhold their consent, whose ages were less than 18 years, who had ongoing pelvic infection, fluid in the pouch of Douglas, and those with previous tubal surgery.

\section{Results}

The mean age for the sampled population was $31.06( \pm 3.96)$ years. The modal parity was the nulliparous group (para 0 ) with $75.2 \%$ of the sampled population in this group. Most (78.2\%) of the women recruited into this study had tertiary education, $19.8 \%$ had secondary education and only $2.0 \%$ had primary education. Most (97\%) of the study participants had secondary infertility while $3 \%$ of the study participants had primary infertility. The duration of infertility was less than two years in $50.5 \%$ of the study participants and was greater than two years in $49.5 \%$ (see Table 1 ).

Concerning uterine fibroids amongst participants of the study, saline SHG agreed with HSG in 15 participants in the diagnosis of uterine fibroid but disagreed in 1 participant. Saline SHG agreed with HSG in the absence of uterine fibroid in 80 of the participants but disagreed in 5 participants. Concordance to diagnosis of uterine fibroids between saline SHG and HSG in this study was $94.06 \%$. There is a statistically significant relationship between saline SHG and HSG in the diagnosis of uterine fibroid $(p=0.0001)$. In this study, saline SHG has a sensitivity of $93.8 \%$, a specificity of $94.1 \%$, Positive Predictive Value of $75 \%$ and Negative Predictive Value of $98.8 \%$ in diagnosis of uterine fibroid when compared to HSG (see Table 2, Figure 1). The Positive Likelihood ratio was 15.94 (95\% CI 6.746 - 37.652) while the Negative Likelihood Ratio was 0.006 (95\% CI $0.010-0.443$ ).

In the diagnosis of uterine adhesions in the participants, Saline SHG agreed with HSG in 5 of the participants in diagnoses of intrauterine adhesion but disagreed in 3 participants. Saline SHG agreed HSG in the absence of intrauterine adhesions in 92 of the participants but disagreed in 1 participant. The concordance to the diagnosis of intrauterine adhesions between saline SHG and HSG in this study was $96.04 \%$. There is a statistically significant relationship between saline SHG and HSG in the diagnosis of intrauterine adhesion as well $(p=0.0001)$. In this study, Saline SHG has a sensitivity of $62.5 \%$, specificity of $98.9 \%$, Positive Predictive Value of $83.3 \%$ and Negative Predictive Value of $96.8 \%$ in the diagnosis of intrauterine adhesions when compared to HSG (see Table 3, Figure 2). The 
Table 1. Socio-demographic characteristic.

\begin{tabular}{|c|c|c|}
\hline VARIABLE & NUMBERS & PERCENTAGES \\
\hline \multicolumn{3}{|l|}{ AGE (years) } \\
\hline $18-25$ & 6 & $5.9 \%$ \\
\hline $26-30$ & 34 & $33.7 \%$ \\
\hline $31-35$ & 53 & $52.5 \%$ \\
\hline$>35$ & 8 & $7.9 \%$ \\
\hline \multicolumn{3}{|c|}{ MARRITAL STATUS } \\
\hline Single & 0 & $0 \%$ \\
\hline Married & 101 & $100 \%$ \\
\hline Divorced & 0 & $0 \%$ \\
\hline Separated & 0 & $0 \%$ \\
\hline \multicolumn{3}{|c|}{ LEVEL OF EDUCATION } \\
\hline Primary & 2 & $2 \%$ \\
\hline Secondary & 20 & $19.8 \%$ \\
\hline Tertiary & 79 & $78.2 \%$ \\
\hline Informal & 0 & $0 \%$ \\
\hline \multicolumn{3}{|l|}{ RELIGION } \\
\hline Christianity & 101 & $100 \%$ \\
\hline Islam & 0 & $0 \%$ \\
\hline others & 0 & $0 \%$ \\
\hline \multicolumn{3}{|l|}{ PARITY } \\
\hline 0 & 76 & $75.2 \%$ \\
\hline $1-4$ & 25 & $24.8 \%$ \\
\hline$>5$ & 0 & $0 \%$ \\
\hline \multicolumn{3}{|c|}{ TYPE OF INFERTILITY } \\
\hline Primary & 3 & $3 \%$ \\
\hline Secondary & 98 & $97 \%$ \\
\hline \multicolumn{3}{|c|}{ DURATION OF INFERTILITY } \\
\hline$<2$ Years & 51 & $50.5 \%$ \\
\hline$>2$ years & 50 & $49.5 \%$ \\
\hline
\end{tabular}

Positive Likelihood ratio was 58.125 (95\% CI 7.969 - 439.018) while the Negative Likelihood Ratio was 0.379 (95\% CI 0.155 - 0.928).

For the diagnosis of patent fallopian tubes, saline SHG agreed with HSG in 86 of the participants in the diagnosis of patent fallopian tube and disagreed in 1 patient. Saline SHG agreed with HSG in blocked fallopian tubes in 11 participants but disagreed in 3 participants. Concordance to a diagnosis of tubal patency between saline SHG and HSG in this study was $96.04 \%$. In this study, 
Table 2. Uterine fibroid with SHG as \% of Uterine fibroid with HSG.

\begin{tabular}{|c|c|c|c|}
\hline SHG HSG & Yes count (\%) & No count (\%) & TOTAL \\
\hline \multirow{2}{*}{ Yes count $(\%)$} & $15(93.8 \%)$ & $5(5.9 \%)$ & \multirow{2}{*}{$20(19.8 \%)$} \\
\hline & TPR or (Sensitivity) & FPR & \\
\hline \multirow{2}{*}{ No count (\%) } & $1(6.2 \%)$ & $80(94.1 \%)$ & \multirow{2}{*}{$81(80.2 \%)$} \\
\hline & FNR & TNR or (Specificity) & \\
\hline Total count (\%) & $16(100 \%)$ & $85(100 \%)$ & $101(100 \%)$ \\
\hline
\end{tabular}

Table 3. Uterine adhesion with SHG as \% uterine adhesion with HSG.

\begin{tabular}{|c|c|c|c|}
\hline SHG HSG & Yes to adhesion (\%) & No to adhesion (\%) & TOTAL \\
\hline \multirow{2}{*}{ Yes to adhesion (\%) } & $5(62.5 \%)$ & $1(1.1 \%)$ & \multirow{2}{*}{$6(5.9 \%)$} \\
\hline & TPR or (Sensitivity) & FPR & \\
\hline \multirow{2}{*}{ No to adhesion (\%) } & $3(37.5 \%)$ & $92(98.9 \%)$ & \multirow{2}{*}{$95(94.1 \%)$} \\
\hline & FNR & TNR or (Specificity) & \\
\hline Total count (\%) & $8(100 \%)$ & $93(100 \%)$ & $101(100 \%)$ \\
\hline
\end{tabular}

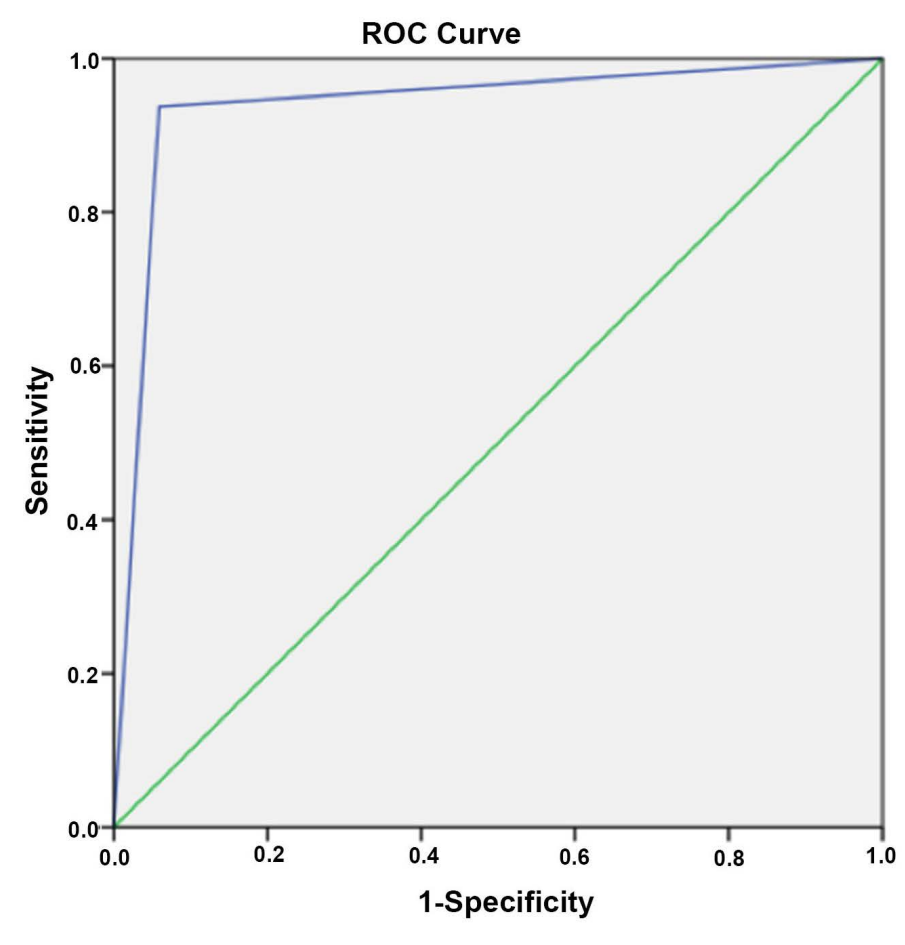

Diagonal segments are produced by ties.

Figure 1. The performance of SHG in diagnosing uterine fibroid using an ROC curve.

saline SHG had a sensitivity of $98.9 \%$ in the diagnosis of patent uterine tubes, specificity of $78.6 \%$, Positive Predictive value of $96.6 \%$ and Negative Predictive value of $91.7 \%$. The False Positive Rate was $21.4 \%$ while the False Negative Rate was $1.1 \%$ when compared to HSG (see Table 4 and Figure 3 ). The Positive Likelihood ratio was 4.613 (95\% CI 1.691 - 12.581) while the Negative Likelihood Ratio was 0.015 (95\% CI $0.002-0.105)$. 


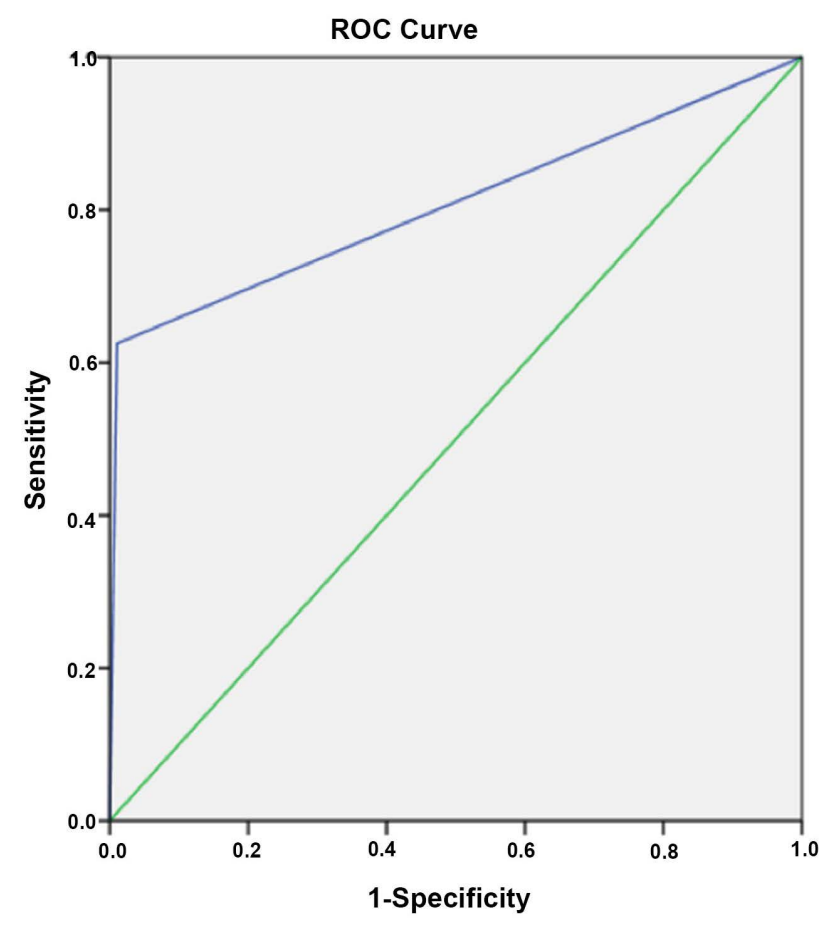

Diagonal segments are produced by ties.

Figure 2. The performance of SHG in diagnosing intrauterine adhesions using an ROC curve.

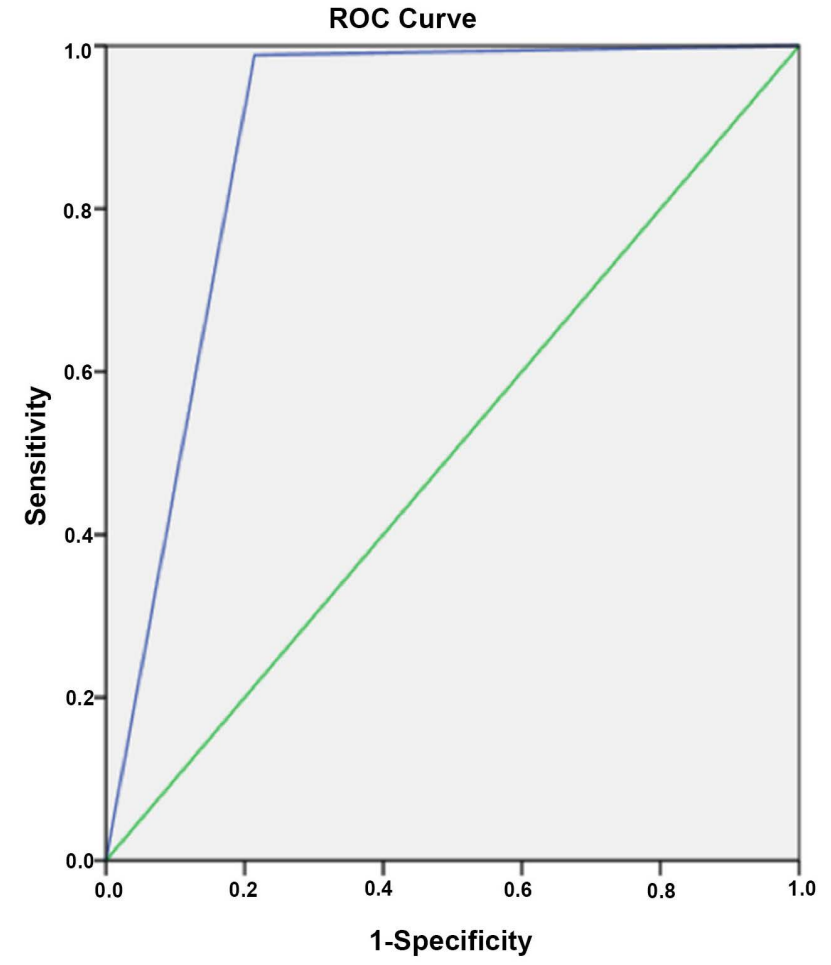

Diagonal segments are produced by ties.

Figure 3. The performance of SHG in the diagnosing of patent tube using an ROC curve. 
Table 4. Patent tubes with SHG and as percentages of patent tubes with HSG.

\begin{tabular}{ccccc}
\hline SHG & HSG & Yes count $(\%)$ & No count $(\%)$ & TOTAL \\
\hline \multirow{2}{*}{ Yes count (\%) } & $86(98.9 \%)$ & $3(21.4 \%)$ & $89(88.1 \%)$ \\
& TPR or (Sensitivity) & FPR & $12(11.9 \%)$ \\
No count (\%) & $1(1.1 \%)$ & $11(78.6 \%)$ & $101(100 \%)$ \\
Total count (\%) & FNR & TNR or (Specificity) & $14(100 \%)$ & $100 \%)$ \\
\hline
\end{tabular}

Abreviations: $\mathrm{TPR}=$ True positive ratio. $\mathrm{TNR}=$ True negative ratio. $\mathrm{FPR}=$ False positive ratio. $\mathrm{FNR}=$ False negative ratio.

\section{Discussion}

The result of this study supports the view that there was a significant agreement between saline SHG and HSG in the diagnosis of uterine fibroid with a concordance rate of $94.06 \%$. This was statistically significant. The sensitivity (93.8\%) and specificity (94.1\%) of saline SHG in the diagnosis of uterine fibroid in this study was higher than the sensitivity (81.8\%) and specificity (93.8\%) of saline SHG in diagnosis of uterine fibroids by Achonolu et al. [14] but lower than the $100 \%$ reported by Goldberg et al. [13] in their study. However, these values still compare favorably to each other. Hysteroscopy was the standard for comparison to saline SHG in the two studies by Acholonu and Goldberg while in this study, saline SHG was compared with HSG. The high sensitivity and specificity of saline SHG in this study and the low False Positive Rate (5.9\%) and False Negative Rate $(6.2 \%)$ indicate that it is a reliable test in the detection or diagnosis of uterine fibroid. The Positive Likelihood Ratio of 15.936 (95\% CI 6.746 - 37.652) as shown in Table 2 and a ROC Curve analysis with AUC of 0.939 (95\% CI 0.865 1.000) as shown in Figure 2 also indicate high accuracy of saline SHG and its usefulness as a clinical tool in the diagnosis of uterine fibroid.

In this study, there was also a high concordance rate of $96.04 \%$ between saline SHG and HSG in the diagnosis of intrauterine adhesions. Though the sensitivity of saline SHG in this study in the diagnosis of intrauterine adhesion was low at $62.5 \%$ when compared to a sensitivity of $75 \%$ and $100 \%$ reported by Soares et al. [17] and Goldberg et al. [13] in their respective studies. This low sensitivity in the diagnosis of intrauterine adhesion in this study implies an increased possibility of saline SHG missing those with intrauterine adhesion. However, saline SHG had a very high specificity of $98.9 \%$ in the diagnosis of intrauterine adhesions in the same study which was comparable to $93 \%$ reported by Soares et al. This implies that saline SHG has a high possibility of identifying women without any intrauterine adhesion. The high Positive Likelihood Ratio of 58.125 (95\% CI 7.696 - 439.018) as shown in Table 3 and the AUC of 0.807 as shown in Figure 2 indicate the high accuracy and clinical usefulness of saline SHG in identifying patients without intrauterine adhesions.

This study showed a concordance rate of $96.04 \%$ between saline SHG and HSG in the diagnosis of tubal patency. This was similar to the findings in the 
study by Spalding et al. [18] which reported a concordance rate of $94 \%$ when saline SHG was compared with HSG. Although this study and that by Spalding et al had comparable agreement rate for saline SHG in the diagnosis of tubal patency and used HSG as the standard of comparison, the reported sensitivity and specificity were quite different. The sensitivity and specificity of saline SHG in this study in the diagnosis of patent tube was very high at $98.9 \%$ and low at $78.6 \%$ respectively when compared to a sensitivity of $50 \%$ and a specificity of $97 \%$ reported by Spalding et al. The disparity in the findings between this study and that by Spalding et al. could be as a result of the difference in sample size, study population and methodology used in their study. Strandell et al. [19] even reported a much lower sensitivity of $27 \%$ for saline SHG in the diagnosis of tubal patency. However, in their study laparoscopy was used as the standard of comparison and was only done for 43 out of 103 study participants. In the study by Strandell et al, saline SHG compared favorably with HSG and they both demonstrated a high concordance with laparoscopy of $83 \%$ and $80 \%$ respectively. Seal et al. reported a comparable sensitivity of $97.3 \%$ for saline SHG in diagnosing tubal patency in their study. A high sensitivity of saline SHG in the diagnosis of tubal patency in this study implies that there is an increased possibility of saline SHG detecting those with patent tube. However, saline SHG had a lower specificity in the diagnosis of tubal patency in this study when compared to $90 \%$ reported by Strandell [17] et al. and $92 \%$ by Seal et al. [15] in their studies. The lower specificity of saline SHG in this study implies that saline SHG has an increased possibility of missing women with tubal blockage in this study. The very low Positive Likelihood Ratio of 4.613 (95\% CI 1.691 - 12.581) indicates a low accuracy of saline SHG in the diagnosis of tubal patency in this study. In this study, an AUC of 0.887 as shown in Figure 3 indicates that saline SHG has a higher chance of diagnosis of tubal patency in those who have patent tubes as in other studies [15] [17] [20].

The summary of findings in this study suggests that saline SHG was comparable to HSG in the detection of uterine fibroid, intrauterine adhesions and tubal patency among infertile women at the University of Port Harcourt Teaching Hospital. Saline SHG more likely to detect uterine fibroids when present or its absence when absent. The likely detection of intrauterine adhesion, if present, with saline SHG was less when compared to the detection of uterine fibroid but its ability to diagnose those without intrauterine adhesions, as those without adhesion, was very high. Saline SHG was also more likely to detect patent fallopian tube when present even better than in the diagnosis of fibroid and uterine adhesions but was less likely to detect those without a patent tube, as such.

\section{Conclusion}

The findings of this study show a significant agreement between results obtained by saline SHG and HSG in the diagnosis of uterine fibroids, intrauterine adhesions and tubal patency among infertile women in the University of Port Harcourt Teaching Hospital. 


\section{Study Limitation}

The study population and power is small. The study was carried out only on infertile patients. We recommend more similar studies with a higher study population to validate this finding in our environment, and in patients without infertility.

\section{Conflicts of Interest}

The authors declare no conflict of interests of any kind in carrying out this study.

\section{References}

[1] Reis, M.M., Soares, S.R., Cancado, M.L. and Camargos, A.F. (1998) Hysterosalpingo-Contrast Sonography (Hycosy) with SH U 454 (Echorist) for the Assessment of Tubal Patency. Human Reproduction, 13, 3049-3052. https://doi.org/10.1093/humrep/13.11.3049

[2] Kumari, R. and Sahay, P. (2018) Comparative Study of Sonohysterosalpingography with Hysterosalpingography for Determination of Tubal Patency in Infertile Women. International Journal of Reproduction, Contraception, Obstetrics and Gynecology, 7, 3117-3121. https://doi.org/10.18203/2320-1770.ijrcog20183301

[3] Allison, S.J., Horrow, M.M., Kim, H.Y. and Lev-Toaft, A.S. (2011) Saline-Infused Sonohysterography: Tips for Achieving Greater Success. Radiographics, 31, 1991-2004. https://doi.org/10.1148/rg.317115074

[4] Panchal, S. and Nagori, C. (2014) Imaging Techniques for Assessment of Tubal Status. Journal of Human Reproductive Sciences, 7, 2-12.

https://doi.org/10.4103/0974-1208.130797

[5] Nwankwo, N.C. and Akani, C.I. (2005) Pattern of Hysterosalpingographic Findings in Infertility in Port Harcourt. West African Journal of Radiology, 12, 15-19. https://doi.org/10.4314/wajr.v12i1.34200

[6] Onwuchekwa, C.R. and Oriji, V.K. (2017) Hysterosalpingographic (HSG) Pattern of Infertility in Women of Reproductive Age. Journal of Human Reproductive Sciences, 10, 178-184. https://doi.org/10.4103/jhrs.JHRS_121_16

[7] Bello, T.O. (2004) Pattern of Tubal Pathology in Infertile Women on Hysterosalpingography in Ilorin, Nigeria. Annals of African Medicine, 3, 77-79.

[8] Okafor, C.O., Okafor, C.I., Okpala, O.C. and Umeh, E. (2010) Pattern of Hysterosalpingographic Finding in Women Being Investigated for Infertility. Nigerian Journal of Clinical Practice, 13, 264-267.

[9] Parson, A.K. and Lense, J.J. (1993) Sonohysterography for Endometrial Abnormalities: Preliminary Results. Journal of Clinical Ultrasound, 21, 87. https://doi.org/10.1002/jcu.1870210203

[10] Tüfekni, E.C., Girit, S., Bayirli, E., Durmupodlu, F. and Yalta, S. (1992) Evaluation of Tubal Patency by Transvaginal Sonosalpingogrphy. Fertility and Sterility, 57, 336-340. https://doi.org/10.1016/S0015-0282(16)54841-9

[11] Nannini, R., Chelo, E., Branconi, F., Tantini, C. and Scarselli, G.F. (1981) Dynamic Echohysteroscopy: A New Diagnostic Technique in the Study Offemale Infertility. Acta Europaea Fertilitatis, 12, 165-171.

[12] Richman, T.S., Viscomi, G.N., de Cherney, A., Polan, M.L. and Alcebo, L.O. (1984) Fallopian Tubal Patency Assessed by Ultrasound Following Fluid Injection. Work 
in Progress. Radiology, 152, 507-510.

https://doi.org/10.1148/radiology.152.2.6539931

[13] Goldberg, J.M., Falcone, T. and Attaran, M. (1997) Sonohysterographic Evaluation of Uterine Abnormalities Noted on Hysterosalpingography. Human Reproduction, 12, 2151-2153. https://doi.org/10.1093/humrep/12.10.2151

[14] Acholonu, U.C., Silberzweig, J., Stein, D.E. and Keltz, M. (2011) Hysterosalpingography versus Sonohysterography for Intrauterine Abnormalities. Journal of the Society of Laparoendoscopic Surgeons, 15, 471-474. https://doi.org/10.4293/108680811X13176785203923

[15] Seal, S.L., Ghosh, D., Saha, D., Bhattacharya, A.R., Ghosh, S. and Mitra, S. (2007) Comparative Evaluation of Sonosalpingography, Hysterosalpingography and Laparoscopy for Determination of Tubal Patency. The Journal of Obstetrics and Gynecology of India, 57, 158-161.

[16] Isreal, G.D. (1992) Determining Sample Size 1. EDIS University of Florida. http://edis.ifas.ufl.edu/pdffiles/pd/pd00600.pdf

[17] Soares, S.R., Barbosa dos Reis, M.M.B. and Camargos, A.F. (2000) Diagnostic Accuracy of Sonohysterography, Transvaginal Sonography and Hysterosalpingography in Patient with Uterine Cavity Diseases. Fertility and Sterility, 73, 406-411. https://doi.org/10.1016/S0015-0282(99)00532-4

[18] Spalding, H., Martikainen, H., Tekay, A. and Jouppila, P. (1999) Transvaginal Salpingosonography for Assessing Tubal Patency in Women with Previously Treated Pelvic Inflammatory Disease and Benign Ovarian Tumours. Ultrasound in Obstetrics \& Gynecology, 14, 205-209. https://doi.org/10.1046/j.1469-0705.1999.14030205.x

[19] Strandell, A., Bourne, T., Bergh, C., Granberg, S., Asztely, M. and Thorburn, J. (1999) The Assessment of Endometrial Pathology and Tubal Patency: A Comparison between the Use of Ultrasonography and X-Ray Hysterosalpingography for the Investigation of Infertility Patients. Ultrasound in Obstetrics \& Gynecology, 14, 200-204. https://doi.org/10.1046/j.1469-0705.1999.14030200.x

[20] Pujar, Y., Sherigar, B., Patted, S., Desai, B.R. and Dhumale, H. (2010) Comparative Evaluation of Saline Infusion Sonohysterography and Hysterolaparoscopy for Diagnosis of Uterine Cavity Abnormalities and Tubal Patency in Infertility: A One Year Cross Sectional Study. JSAFOG, 2, 133-135.

https://doi.org/10.5005/jp-journals-10006-1081 